\title{
MULTIPLE SOLUTIONS FOR NONLINEAR DISCONTINUOUS STRONGLY RESONANT ELLIPTIC PROBLEMS
}

\author{
NIKOLAOS C. KOUROGENIS AND NIKOLAOS S. PAPAGEORGIOU
}

Received 22 August 1999

We consider quasilinear strongly resonant problems with discontinuous right-hand side. To develop an existence theory we pass to a multivalued problem by, roughly speaking, filling in the gaps at the discontinuity points. We prove the existence of at least three nontrivial solutions. Our approach uses the nonsmooth critical point theory for locally Lipschitz functionals due to Chang (1981) and a generalized version of the Ekeland variational principle. At the end of the paper we show that the nonsmooth Palais-Smale (PS)-condition implies the coercivity of the functional, extending this way a well-known result of the "smooth" case.

\section{Introduction}

In $[16,17]$, we studied quasilinear elliptic problems at resonance and near resonance with discontinuous right-hand side. In [16], we investigated the resonant problem and using a variational approach, we proved the existence of a nontrivial solution. In [17], we considered problems near resonance with the parameter $\lambda$ approaching from the left the first eigenvalue $\lambda_{1}$ of the $p$-Laplacian. For such problems we prove the existence of at least three nontrivial solutions. At the end of that paper, we mention, as an open problem, the existence of multiple nontrivial solutions for the resonant equation. The aim of this work is to give a solution to this open problem. Contrary to what we had in [16], here we assume that the potential function $F(z, x)=\int_{0}^{x} f(z, r) d r$ has a finite limit for a.a. $z \in \mathbb{Z}$ as $x \rightarrow \pm \infty$. In this respect our work is similar to that of Thews [21], Bartolo et al. [8], and Ward [23]. In [8], this case was termed "strongly resonant." The case where $\lim _{x \rightarrow \pm \infty} F(z, x)$ is infinite for all $z \in E_{ \pm}$with $\left|E_{ \pm}\right|>0$ (here we denote by $|\cdot|$ the Lebesgue measure on $\mathbb{R}^{N}$ ) was considered by Ahmad et al. [3] and Rabinowitz [19] while a mixed situation with $\lim _{x \rightarrow \infty} F(x)=0$ and $\lim _{x \rightarrow-\infty} F(x)=+\infty$ was studied by Costa and Silva [12]. All the aforementioned works deal with semilinear equations which have a continuous right-hand side and prove the existence of one nontrivial solution. Multiple solutions for semilinear resonant 
problems with a continuous $f$, were proved by Ahmad [2], Goncalves and Miyagaki $[13,14]$, and Landesman et al. [18]. The first multiplicity result for the quasilinear resonant problem was obtained recently by Alves et al. [4], who studied an equation with the $p$-Laplacian and a continuous right-hand side. To our knowledge, our theorem is the first multiplicity result for strongly resonant quasilinear problems with discontinuities. We hope that our work here will motivate further research on the problem which will improve our result by relaxing some of our hypotheses and by obtaining new multiplicity results.

Our approach combines the critical point theory for nonsmooth locally Lipschitz functionals due to Chang [10] with a recently obtained extension of the Ekeland variational principle due to Zhong [24]. We make use of a weak form of the nonsmooth Palais-Smale condition, originally due to [9] (for smooth problems) which has been exploited by Bartolo et al. [8] (again in the context of smooth problems). We show that the quasilinear discontinuous resonant problem has at least three nontrivial solutions. In Section 4, we show that the usual nonsmooth Palais-Smale condition, introduced by Chang [10] implies the coercivity of the functionals. This allows us to extend the well-known result in the "smooth" context to the present case.

\section{Preliminaries}

Let $X$ be a Banach space and let $X^{*}$ be its topological dual. A function $f: X \rightarrow \mathbb{R}$ is said to be "locally Lipschitz," if for every $x \in X$ there exists a neighbourhood $U$ of $x$ and a constant $k>0$ depending on $U$ such that $|f(z)-f(y)| \leq k\|z-y\|$ for all $z, y \in U$. From convex analysis we know that a proper, convex, and lower semicontinuous function $g: X \rightarrow \overline{\mathbb{R}}=\mathbb{R} \cup\{+\infty\}$ is locally Lipschitz in the interior of its effective domain dom $g=\{x \in X: g(x)<+\infty\}$. For a locally Lipschitz $f$, we define the "generalized directional derivative" at $x \in X$ in the direction $h \in X$ by

$$
f^{0}(x ; h)=\varlimsup_{\substack{x^{\prime} \rightarrow x \\ \lambda \downarrow 0}} \frac{f\left(x^{\prime}+\lambda h\right)-f\left(x^{\prime}\right)}{\lambda} .
$$

It is easy to check that $h \rightarrow f^{0}(x ; h)$ is sublinear, continuous (in fact $\left|f^{0}(x ; h)\right| \leq$ $k\|h\|$, hence $f^{0}(x ; \cdot)$ is Lipschitz continuous). So from the Hahn-Banach theorem, we know that $f^{0}(x ; \cdot)$ is the support function of a nonempty, convex and $w^{*}$-compact set defined by

$$
\partial f(x)=\left\{x^{*} \in X^{*}:\left(x^{*}, h\right) \leq f^{0}(x ; h) \forall h \in X\right\} .
$$

The set $\partial f(x)$ is called the "generalized (or Clarke) subdifferential" of $f(\cdot)$ at $x$. If $f, g: X \rightarrow \mathbb{R}$ are locally Lipschitz functions, then $\partial(f+g)(x) \subseteq \partial f(x)+\partial g(x)$ and $\partial(\lambda f)(x)=\lambda \partial f(x)$ for all $\lambda \in \mathbb{R}$. Moreover, if $f: X \rightarrow \mathbb{R}$ is also convex, then the generalized subdifferential and the subdifferential in the sense of convex analysis coincide. If $f$ is strictly differentiable at $x$ (in particular, if $f$ is continuously Gateaux differentiable at $x$ ), then $\partial f(x)=\left\{f^{\prime}(x)\right\}$. A point $x \in X$ is a "critical point" of $f$ if $0 \in \partial f(x)$. It is easy to see that if $x \in X$ is a local minimum or maximum, then $0 \in \partial f(x)$. For details we refer to Clarke [11]. 
It is well known that the classical critical point theory for smooth functions, uses a compactness condition known as the "Palais-Smale condition" ((PS)-condition). In the present nonsmooth context this condition takes the following form: we say that $f$ satisfies the "nonsmooth (PS)-condition," if any sequence $\left\{x_{n}\right\}_{n \geq 1} \subseteq X$ along which $\left\{f\left(x_{n}\right)\right\}_{n \geq 1}$ is bounded and $m\left(x_{n}\right)=\min \left\{\left\|x^{*}\right\|: x^{*} \in \partial f\left(x_{n}\right)\right\} \stackrel{n \rightarrow \infty}{\longrightarrow} 0$, has a strongly convergent subsequence. If $f \in C^{1}(X)$, then since $\partial f\left(x_{n}\right)=\left\{f^{\prime}\left(x_{n}\right)\right\}$, we see that the above definition coincides with the classical (PS)-condition (see Rabinowitz [19]).

A weaker form of the (PS)-condition was introduced for smooth functions by Cerami [9]. Cerami's condition for a locally Lipschitz functional $R: X \rightarrow \mathbb{R}$ in the present nonsmooth setting has the following form: "Any sequence $\left\{x_{n}\right\}_{n \geq 1} \subseteq X$ such that $\left|R\left(x_{n}\right)\right| \leq M, n \geq 1$, and $\left(1+\left\|x_{n}\right\|\right) m\left(x_{n}\right) \stackrel{n \rightarrow \infty}{\longrightarrow} 0$, has a strongly convergent subsequence." We call this condition "nonsmooth $C$-condition." It was proved in the smooth case by Bartolo et al. [8, Theorem 1.3], that this weaker compactness condition suffices in order to have the deformation theorem and from that derive minimax principles. The same can be done in the nonsmooth case, where we can obtain the deformation theorem of Chang [10, Theorem 3.1] by simple modifications of the proof of Bartolo et al. [8] based on Chang [10, Lemmas 3.1-3.4], and then have the nonsmooth minimax principles. An alternative approach, avoiding the deformation theorem, can be based in the recent generalization of the Ekeland variational principle due to Zhong [24] (see also Theorem 2.2 below). Evidently the nonsmooth (PS)-condition implies the nonsmooth $C$-condition. We say that a locally Lipschitz functional $R: X \rightarrow \mathbb{R}$ satisfies the "nonsmooth $C$-condition at level $c$ " (respectively, the "nonsmooth (PS)condition at level $c$ "), if any sequence $\left\{x_{n}\right\}_{n \geq 1} \subseteq X$ satisfying $R\left(x_{n}\right) \stackrel{n \rightarrow \infty}{\longrightarrow} c$ and $\left(1+\left\|x_{n}\right\|\right) m\left(x_{n}\right) \stackrel{n \rightarrow \infty}{\longrightarrow} 0$ (respectively, $m\left(x_{n}\right) \stackrel{n \rightarrow \infty}{\longrightarrow} 0$ ) has a convergent subsequence. If these are true for every $c \in \mathbb{R}$, then we have the previously introduced "global" definitions.

The next theorem is due to Chang [10] and extends to a nonsmooth setting the well-known "mountain pass theorem" due to Ambrosetti and Rabinowitz [5].

Theorem 2.1. If $X$ is a reflexive Banach space, $R: X \rightarrow \mathbb{R}$ is locally Lipschitz, there exist $y \in X$ and $r>0$ with $\|y\|>r$ such that $\max \{R(0), R(y)\}<\inf [R(x):\|x\|=r]$, $c=\inf _{\Gamma} \max _{0 \leq t \leq 1} R(\gamma(t))$, where $\Gamma=\{\gamma \in C([0,1], X): \gamma(0)=0, \gamma(1)=y\}$ and $R(\cdot)$ satisfies the nonsmooth $C$-condition at level $c$, then $c \geq \inf [R(x):\|x\|=r]$ and there exists $x \in X$ such that $0 \in \partial R(x), R(x)=c$.

Let $Z \subseteq \mathbb{R}^{N}$ be a bounded domain. Consider the following nonlinear eigenvalue problem:

$$
-\operatorname{div}\left(\|D x(z)\|^{p-2} D x(z)\right)=\lambda|x(z)|^{p-2} x(z) \quad \text { a.e. on } Z,\left.x\right|_{\Gamma}=0 .
$$

The least real number for which problem (2.3) has a nontrivial solution, is the first eigenvalue of the negative $p$-Laplacian $-\triangle_{p} x=-\operatorname{div}\left(\|D x\|^{p-2} D x\right)$ with Dirichlet boundary conditions (i.e., of $\left.\left(-\triangle_{p}, W_{0}^{1, p}(Z)\right)\right)$ and is denoted by $\lambda_{1}$. This first eigenvalue $\lambda_{1}$ 
is positive, isolated, and simple (i.e., the associated eigenfunctions are constant multiples of each other). Moreover, we have the following variational characterization of $\lambda_{1}>0$ via a Rayleigh quotient, namely

$$
\lambda_{1}=\min \left[\frac{\|D x\|_{p}^{p}}{\|x\|_{p}^{p}}: x \in W_{0}^{1, p}(Z), x \neq 0\right] .
$$

This minimum is realized at the normalized eigenfunction $u_{1}$. Note that if $u_{1}$ minimizes the Rayleigh quotient, then so does $\left|u_{1}\right|$ and so we infer that the first eigenfunction $u_{1}$ does not change sign on $Z$. In fact we can show that $u_{1} \neq 0$ a.e. on $Z$ and so we may assume that $u_{1}(z)>0$ a.e. on $Z$ (note that when the boundary of $Z$ is smooth, by nonlinear elliptic regularity theory, $u \in C_{\mathrm{loc}}^{1, \beta}(Z), 0<\beta<1$; see Tolksdorf [22]). The first work on the properties of $\lambda_{1}$ when $Z \subseteq \mathbb{R}^{N}$ is a bounded domain of Holder class $C^{2, a}$ was obtained by Anane [6]. His result was extended to general bounded domains by Lindqvist [21].

The Ljusternik-Schnirelmann theory gives, in addition to $\lambda_{1}$, a whole strictly increasing sequence of positive real numbers $0<\lambda_{1}<\lambda_{2}<\lambda_{3}<\cdots<\lambda_{k}<\cdots$ for which there exist nontrivial solutions of the nonlinear eigenvalue problem (2.3). In other words, the spectrum $\sigma\left(-\triangle_{p}\right)$ of $\left(-\triangle_{p}, W_{0}^{1, p}(Z)\right)$ contains at least these points $\left\{\lambda_{k}\right\}_{k \geq 1}$. Nothing is known about the possible existence of other points in $\sigma\left(-\triangle_{p}\right) \subseteq\left[\lambda_{1}, \infty\right) \subseteq \mathbb{R}_{+}$. However, if $X=\left\langle u_{1}\right\rangle=\mathbb{R} u_{1}$ and $V$ is a topological complement (i.e., $\left.W_{0}^{1, p}(Z)=X \oplus V\right)$, then because $\lambda_{1}>0$ is isolated we have

$$
\hat{\lambda}_{2 V}=\inf \left[\frac{\|D v\|_{p}^{p}}{\|v\|_{p}^{p}}: v \in V, v \neq 0\right]>\lambda_{1}, \quad \hat{\lambda}_{2}=\sup _{V} \hat{\lambda}_{2 V}
$$

Concerning $\hat{\lambda}_{2}$ we have the important recent work of Anane and Tsouli [7], who proved that $\hat{\lambda}_{2}$ is the second eigenvalue of $\left(-\triangle_{p}, W_{o}^{1, p}(Z)\right)$. Finally, we recall the following generalization of the Ekeland variational principle due to Zhong [24] which we will need in what follows.

TheOREM 2.2. If $h: \mathbb{R}_{+} \rightarrow \mathbb{R}_{+}$is a continuous nondecreasing function such that $\int_{0}^{\infty} 1 /(1+h(r)) d r=+\infty,(Y, d)$ is a complete metric space, $x_{0} \in Y$ is fixed, $\phi$ : $Y \rightarrow \overline{\mathbb{R}}=\mathbb{R} \cup\{+\infty\}$ is a lower semicontinuous function not identically $+\infty$ which is bounded from below then for any given $\lambda>0, \varepsilon>0$, and $y \in Y$ such that $\phi(y) \leq$ $\inf _{Y} \phi+\varepsilon$, we can find $x \in Y$ such that

(a) $\phi(x) \leq \phi(y)$;

(b) $\phi(x) \leq\left(\phi(u)+\varepsilon /\left(\lambda\left(1+h\left(d\left(x_{0}, x\right)\right)\right)\right)\right) d(u, x)$ for all $u \in Y$; and

(c) $d\left(x, x_{0}\right) \leq d\left(y, x_{0}\right)+\bar{r}$, where $\bar{r}>0$ is such that $\int_{d\left(y, x_{0}\right)}^{d\left(y, x_{0}\right)+\bar{r}} 1 /(1+h(r)) d r$ $\geq \lambda$.

Remark 2.3. If $h(r) \equiv 0$ and $x_{0}=y$, then we recover Ekeland's variational principle (cf. Hu and Papageorgiou [15]). 
Now we are ready to start studying our problem. So let $Z \subseteq \mathbb{R}^{N}$ be a bounded domain with a $C^{1}$-boundary $\Gamma$. We consider the following quasilinear resonant problem:

$$
\begin{aligned}
-\operatorname{div}\left(\|D x(z)\|^{p-2} D x(z)\right)-\lambda_{1}|x(z)|^{p-2} x(z) & =f(z, x(z)) \\
\text { a.e. on } Z,\left.x\right|_{\Gamma} & =0,2 \leq p<\infty .
\end{aligned}
$$

We do not assume that $f(z, \cdot)$ is continuous and so problem (2.6) need not have a solution. To be able to develop a reasonable existence theory, we pass to a multivalued version of (2.6) by, roughly speaking, filling in the gaps at the discontinuity points of $f(z, \cdot)$. More precisely, we introduce the following two functions:

$$
\begin{aligned}
& f_{1}(z, x)={\underset{x \rightarrow x}{x^{\prime} \rightarrow x}}_{\lim } f\left(z, x^{\prime}\right)=\lim _{\delta \downarrow 0} \underset{\left|x^{\prime}-x\right|<\delta}{\operatorname{essinf}} f\left(z, x^{\prime}\right), \\
& f_{2}(z, x)=\varlimsup_{x^{\prime} \rightarrow x} f\left(z, x^{\prime}\right)=\lim _{\delta \downarrow 0} \operatorname{ess} \sup _{\left|x^{\prime}-x\right|<\delta} f\left(z, x^{\prime}\right) .
\end{aligned}
$$

Using them we define the multifunction $\hat{f}(z, x)=\left\{y \in \mathbb{R}: f_{1}(z, x) \leq y \leq f_{2}(z, x)\right\}$. Then instead of (2.6) we study the following quasilinear resonant elliptic inclusion:

$$
\begin{array}{r}
-\operatorname{div}\left(\|D x(z)\|^{p-2} D x(z)\right)-\lambda_{1}|x(z)|^{p-2} x(z) \in \hat{f}(z, x(z)) \\
\text { a.e. on } Z,\left.x\right|_{\Gamma}=0,2 \leq p<\infty .
\end{array}
$$

By a solution of (2.8) we mean a function $x \in W_{0}^{1, p}(Z)$ such that $\operatorname{div}\left(\|D x(\cdot)\|^{p-2} D x(\cdot)\right)$ $\in L^{1}(Z)$ and $-\operatorname{div}\left(\|D x(z)\|^{p-2} D x(z)\right)-\lambda_{1}|x(z)|^{p-2} x(z)=u(z)$ a.e. on $Z$ with $u \in L^{1}(Z), f_{1}(z, x(z)) \leq u(z) \leq f_{2}(z, x(z))$ a.e. on $Z$. We show that (2.8) has at least three distinct nontrivial solutions. For this purpose we introduce the following hypotheses on $f(z, x)$.

(H) $f: Z \times \mathbb{R} \rightarrow \mathbb{R}$ is a measurable function such that

(i) $f_{1}, f_{2}$ are $N$-measurable functions (i.e., for every $x: Z \rightarrow \mathbb{R}$ measurable function, $z \rightarrow f_{1}(z, x(z))$ and $z \rightarrow f_{2}(z, x(z))$ are measurable functions; superpositional measurability);

(ii)

$$
|f(z, x)| \leq \begin{cases}a_{1}(z) & \text { for a.a. } z \in Z, \text { all } x<0 \\ a_{2}(z)+c_{2}|x|^{\sigma-1} & \text { for a.a. } z \in Z, \text { all } x \geq 0\end{cases}
$$

where $a_{1}, a_{2} \in L^{\infty}(Z), c_{2}>0$, and $1 \leq \sigma \leq p^{*}, p^{*}=N_{p} /(N-p)$;

(iii) there exist $F_{ \pm} \in L^{1}(Z)$ such that $F(z, x) \stackrel{x \rightarrow-\infty}{\longrightarrow} F_{-}(z)$ and $F(z, x) \stackrel{x \rightarrow \infty}{\longrightarrow}$ $F_{+}(z)$ uniformly for a.a. $z \in Z$

(iv) for a.a. $z \in Z$ and all $x \in \mathbb{R}, p F(z, x) \leq\left(\hat{\lambda}_{2}-\lambda_{1}\right)|x|^{p}$;

(v) there exist constants $\eta, \gamma>0$ such that for a.a. $z \in Z$ and all $x \geq \eta>0$ we have $\left(x u^{*}-p F(z, x)\right) /\left|u^{*}\right|^{1+1 / r} \geq \gamma>0$ for all $u^{*} \in \hat{f}(z, x)$ and with 


$$
\begin{aligned}
& 1 \leq r \leq p^{*}-1 \text {. Further if }\left\{x_{n}\right\}_{n \geq 1} \subseteq W_{0}^{1, p}(Z) \text { is such that }\left|x_{n}(z)\right| \stackrel{n \rightarrow \infty}{\longrightarrow} \infty \\
& \text { a.e. on } Z \text {, then } \int_{Z} f_{i}\left(z, x_{n}(z)\right) x_{n}(z) d z \stackrel{x \rightarrow \infty}{\longrightarrow} 0 \text { for } i=1,2 ;
\end{aligned}
$$

(vi) $\varlimsup_{x \rightarrow 0}\left(p F(z, x) /|x|^{p}\right)<-\lambda_{1}$ uniformly for a.a. $z \in Z$;

(vii) there exist $\xi_{-}<0<\xi_{+}$such that $\int_{Z} F\left(z, \xi_{ \pm} u_{1}(z)\right) d z>0$ and $\int_{Z} F(z$, $\left.\xi_{ \pm} u_{1}(z)\right) d z>\int_{Z} F_{ \pm}(z) d z$.

Remark 2.4. Hypothesis (H)(i) is satisfied if $f$ is independent of $z \in Z$ or if for a.a. $z \in Z, f(z, \cdot)$ is monotone nondecreasing. Indeed, in the first case the $N$-measurability of $f_{1}$ and $f_{2}$ follows from the fact that $f_{1}$ is lower semicontinuous, while $f_{2}$ is upper semicontinuous. In the second case note that $f_{1}(z, x)=\lim _{n \rightarrow \infty} f(z, x-1 / n)$ and $f_{2}(z, x)=\lim _{n \rightarrow \infty} f(z, x+1 / n)$, hence both functions $f_{1}$ and $f_{2}$ are measurable, thus $N$-measurable too. Hypothesis (H)(iii) is the "strong resonance" condition since for a.a. $z \in Z, F_{ \pm}$are finite. Evidently by virtue of hypothesis $(\mathrm{H})(\mathrm{vi})$, the growth condition imposed in (H)(iv) is automatically satisfied in a neighbourhood of zero. Note that hypothesis (H)(iv) is analogous to hypothesis $H_{\infty}$ of Goncalves and Miyagaki [14] and hypothesis ( $\left.g_{5}\right)$ of Costa and Silva [12]. The first part of hypothesis $(\mathrm{H})(\mathrm{v})$ is a variant of the well-known Ambrosetti and Rabinowitz condition (see Ambrosetti and Rabinowitz [5] or Rabinowitz [19]). It is consistent with hypothesis (H)(ii), (iii), (iv) and it implies that for $x \geq \eta>0$ we have $F(z, x)<0$. If for $i=1,2, f_{i}(z, x) x \stackrel{|x| \rightarrow \infty}{\longrightarrow} 0$ uniformly for all $z \in Z$, then the second part of hypothesis $(\mathrm{H})(\mathrm{v})$ is satisfied. Because of hypothesis $(\mathrm{H})(\mathrm{vii})$, we do not need the second part of hypothesis $(\mathrm{H})(\mathrm{v})$ for the proof of the existence of two solutions. Hypothesis $(\mathrm{H})(\mathrm{vi})$ is needed in order to be able to apply Theorem 2.1 and have a third nontrivial solution. Without it we cannot guarantee that the third solution (which in this case is obtained via the mountain pass theorem) is nontrivial.

\section{Auxiliary results}

Let $R: W_{0}^{1, p}(Z) \rightarrow \mathbb{R}$ be the energy functional defined by

$$
R(x)=\frac{1}{p}\|D x\|_{p}^{p}-\frac{\lambda_{1}}{p}\|x\|_{p}^{p}-\int_{Z} F(z, x(z)) d z .
$$

Because of hypothesis $(\mathrm{H})(\mathrm{iii})$ and since $W_{0}^{1, p}(Z)$ is embedded continuously in $L^{\sigma}(Z)$ (Sobolev embedding theorem), from Chang [10] we have that $R(\cdot)$ is locally Lipschitz. In this section, we prove a series of auxiliary results which determine the properties of $R(\cdot)$.

Proposition 3.1. If hypotheses $(\mathrm{H})$ hold, then $R(\cdot)$ is bounded below.

Proof. Using hypothesis (H)(iii), we can find $M>0$ such that for a.a. $z \in Z$ we have

$$
\begin{gathered}
\left|F(z, x)-F_{-}(z)\right| \leq 1 \quad \forall x \leq-M, \\
\left|F(z, x)-F_{+}(x)\right| \leq 1 \quad \forall x \geq M .
\end{gathered}
$$


Also by virtue of (H)(ii), we see that for a.a. $z \in Z$ and all $|x|<M,|F(z, x)| \leq \hat{a}(z)$ with $\hat{a} \in L^{\infty}(Z)$. Then for every $x \in W_{0}^{1, p}(Z)$ we have

$$
\begin{aligned}
R(x)= & \frac{1}{p}\|D x\|_{p}^{p}-\frac{\lambda_{1}}{p}\|x\|_{p}^{p}-\int_{\{|x(z)|<M\}} F(z, x(z)) d z \\
& -\int_{\{x(z)<-M\}} F(z, x(z)) d z-\int_{\{x(z)>M\}} F(z, x(z)) d z \\
\geq & -\|\hat{a}\|_{1}-\left\|F_{-}\right\|_{1}-\left\|F_{+}\right\|_{1}-2|Z| .
\end{aligned}
$$

Recall that $W_{0}^{1, p}(Z)=X \oplus V$ with $X=\left\langle u_{1}\right\rangle=\mathbb{R} u_{1}$ and $V$ a topological complement.

Proposition 3.2. If hypotheses $(H)$ hold, then $\left.R\right|_{V} \geq 0$.

Proof. Using hypothesis (H)(iv) and (2.5), for every $v \in V$ we have

$$
\begin{aligned}
R(v) & =\frac{1}{p}\|D v\|_{p}^{p}-\frac{\lambda_{1}}{p}\|v\|_{p}^{p}-\int_{Z} F(z, v(z)) d z \\
& \geq \frac{1}{p}\|D v\|_{p}^{p}-\frac{\lambda_{1}}{p}\|v\|_{p}^{p}-\frac{1}{p}\left(\hat{\lambda}_{2}-\lambda_{1}\right)\|v\|_{p}^{p} \\
& \geq \frac{1}{p}\|D v\|_{p}^{p}-\frac{\lambda_{1}}{p}\|v\|_{p}^{p}-\frac{1}{p}\|D v\|_{p}^{p}+\frac{\lambda_{1}}{p}\|v\|_{p}^{p}=0 .
\end{aligned}
$$

Recalling that $\left\|D u_{1}\right\|_{p}^{p}=\lambda_{1}\left\|u_{1}\right\|_{p}^{p}$ (see Section 2) and using hypothesis (H)(vii), we have the following propositions.

Proposition 3.3. If hypotheses $(H)$ hold, then $R\left(\xi_{ \pm} u_{1}\right)<0$.

Proposition 3.4. If hypotheses $(H)$ hold, then $R(\cdot)$ satisfies the nonsmooth $C$-condition at level $c \neq-\int_{Z} F_{ \pm}(z) d z$.

Proof. Let $\left\{x_{n}\right\}_{n \geq 1} \subseteq W_{0}^{1, p}(Z)$ be a sequence such that $R\left(x_{n}\right) \stackrel{n \rightarrow \infty}{\longrightarrow} c, c \neq-\int_{Z} F_{ \pm}(z) d z$ and $\left(1+\left\|x_{n}\right\|_{1, p}\right) m\left(x_{n}\right) \stackrel{n \rightarrow \infty}{\longrightarrow} 0$ where for every $x \in W_{0}^{1, p}(Z), m(x)=\inf \left\{\left\|x^{*}\right\|: x^{*} \in\right.$ $\partial R(x)\}$ (see Section 2). Let $x_{n}^{*} \in \partial R\left(x_{n}\right), n \geq 1$, be such that $\left\|x_{n}^{*}\right\|=m\left(x_{n}\right)$. Its existence follows from the fact that $\partial R\left(x_{n}\right)$ is weakly compact in $W^{-1, q}(Z)$ and from the weak lower semicontinuity of the norm functional. Since $x_{n}^{*} \in \partial R\left(x_{n}\right)$, we have

$$
x_{n}^{*}=A\left(x_{n}\right)-\lambda_{1} J\left(x_{n}\right)-u_{n}^{*}, \quad n \geq 1,
$$

where $A: W_{0}^{1, p}(Z) \rightarrow W^{-1, q}(Z)(1 / p+1 / q=1)$ is defined by $\langle A(x), y\rangle=$ $\int_{Z}\|D x(z)\|^{p-2}(D x(z), D y(z))_{\mathbb{R}^{N}} d z, J\left(x_{n}\right)(\cdot)=\left|x_{n}(\cdot)\right|^{p-2} x_{n}(\cdot)$, and $u_{n}^{*} \in \partial \psi\left(x_{n}\right)$, where $\psi(x)=\int_{Z} F(z, x(z)) d z$ for all $x \in W_{0}^{1, p}(Z)$. Note that by $\langle\cdot, \cdot\rangle$ we denote the duality brackets for the pair $\left(W_{0}^{1, p}(Z), W^{-1, q}(Z)\right)$. From Chang [10] we know that $f_{1}\left(z, x_{n}(z)\right) \leq u_{n}^{*}(z) \leq f_{2}\left(z, x_{n}(z)\right)$ a.e. on $Z$. Also it is easy to check that $A$ is monotone, demicontinuous, hence maximal monotone (see Hu and Papageorgiou [15]). 
Claim. $\left\{x_{n}\right\}_{n \geq 1} \subseteq W_{0}^{1, p}(Z)$ is bounded.

Suppose not. Then by passing to a subsequence if necessary, we may assume that $\left\|x_{n}\right\| \stackrel{n \rightarrow \infty}{\longrightarrow} \infty,\left\|x_{n}\right\| \neq 0$ for all $n \geq 1$. From the choice of the sequence $\left\{x_{n}\right\}_{n \geq 1}$, we have

$$
\begin{gathered}
p R\left(x_{n}\right)=\left\|D x_{n}\right\|_{p}^{p}-\lambda_{1}\left\|x_{n}\right\|_{p}^{p}-p \psi\left(x_{n}\right) \leq p M_{1} \quad \text { for some } M_{1}>0, \\
\left|\left\langle x_{n}^{*}, x_{n}\right\rangle\right| \leq \varepsilon_{n} \quad \text { with } \varepsilon_{n} \downarrow 0 .
\end{gathered}
$$

From (3.7) we have

$$
-\left\langle A\left(x_{n}\right), x_{n}\right\rangle+\lambda_{1}\left(J\left(x_{n}\right), x_{n}\right)_{q p}+\left(u_{n}^{*}, x_{n}\right)_{\sigma^{\prime} \sigma} \leq \varepsilon_{n},
$$

where by $(\cdot, \cdot)_{q p}$ we denote the duality brackets for the pair $\left(L^{q}(Z), L^{p}(Z)\right), 1 / p+$ $1 / q=1$ and by $(\cdot, \cdot)_{\sigma^{\prime} \sigma}$ the duality brackets for the pair $\left(L^{\sigma^{\prime}}(Z), L^{\sigma}(Z)\right), 1 / \sigma+1 / \sigma^{\prime}$ $=1$. Note that by the Sobolev embedding theorem $W_{0}^{1, p}(Z)$ is embedded continuously in $L^{p}(Z)$ and in $L^{\sigma}(Z)$, hence $x_{n} \in L^{p}(Z) \cap L^{\sigma}(Z)$ for all $n \geq 1$, while $J\left(x_{n}\right) \in L^{q}(Z)$ and $u_{n}^{*} \in L^{\sigma^{\prime}}(Z)$ (see hypothesis (H)(iii)). So we have

$$
-\left\|D x_{n}\right\|_{p}^{p}+\lambda_{1}\left\|x_{n}\right\|_{p}^{p}+\int_{Z} u_{n}^{*}(z) x_{n}(z) d z \leq \varepsilon_{n} .
$$

Adding (3.6) and (3.9), we obtain

$$
\begin{gathered}
-p \psi\left(x_{n}\right)+\int_{Z} u_{n}^{*}(z) x_{n}(z) d z \leq p M_{1}+c_{3} \quad \text { for some } c_{3}>0, \\
\Longrightarrow \int_{\left\{x_{n}<0\right\}}\left(u_{n}^{*}(z) x_{n}(z)-p F\left(z, x_{n}(z)\right)\right) d z \\
\quad+\int_{\left\{x_{n} \geq 0\right\}}\left(u_{n}^{*}(z) x_{n}(z)-p F\left(z, x_{n}(z)\right)\right) d z \leq p M_{1}+c_{3} .
\end{gathered}
$$

Because of hypothesis $(\mathrm{H})(\mathrm{ii})$, we have

$$
\left|\int_{\left\{x_{n}<0\right\}}\left(u_{n}^{*}(z) x_{n}(z)-p F\left(z, x_{n}(z)\right)\right) d z\right| \leq c_{4}\left\|x_{n}\right\|_{1, p} \quad \text { for some } c_{4}>0 .
$$

Using (3.11) in (3.10), we obtain

$$
\begin{aligned}
\int_{\left\{x_{n} \geq 0\right\}}\left(u_{n}^{*}(z) x_{n}(z)\right. & \left.-p F\left(z, x_{n}(z)\right)\right) d z \\
& \leq c_{5}+c_{4}\left\|x_{n}\right\|_{1, p} \quad\left(\text { with } c_{5}=p M_{1}+c_{3}\right) .
\end{aligned}
$$

Dividing by $\left\|x_{n}\right\|_{1, p}^{1+1 / r}$, we have

$$
\begin{gathered}
\int_{\left\{x_{n} \geq 0\right\}} \frac{u_{n}^{*}(z) x_{n}(z)-p F\left(z, x_{n}(z)\right)}{\left\|x_{n}\right\|_{1, p}^{1+1 / r}} d z \leq \frac{c_{5}}{\left\|x_{n}\right\|_{1, p}^{1+1 / r}}+\frac{c_{4}}{\left\|x_{n}\right\|_{1, p}^{1 / r}} \\
\Longrightarrow \lim _{n \rightarrow \infty} \int_{\left\{x_{n} \geq 0\right\}} \frac{u_{n}^{*}(z) x_{n}(z)-p F\left(z, x_{n}(z)\right)}{\left\|x_{n}\right\|_{1, p}^{1+1 / r}} d z=0 .
\end{gathered}
$$


Note that in concluding the last equality (and not only the inequality $\leq 0$ ), we have used hypothesis $(\mathrm{H})(\mathrm{v})$. Also from the same hypothesis, we see that for a.a. $z \in Z$, if $x_{n}(z) \geq \eta>0$, then $u_{n}^{*}(z) \neq 0$. So we can write that

$$
\begin{aligned}
\int_{\left\{0 \leq x_{n}\right\}} \frac{u_{n}^{*}(z) x_{n}(z)-p F\left(z, x_{n}(z)\right)}{\left\|x_{n}\right\|_{1, p}^{1+1 / r}} d z= & \int_{\left\{0 \leq x_{n}<\eta\right\}} \frac{u_{n}^{*}(z) x_{n}(z)-p F\left(z, x_{n}(z)\right)}{\left\|x_{n}\right\|_{1, p}^{1+1 / r}} d z \\
& +\int_{\left\{x_{n} \geq \eta\right\}} \frac{u_{n}^{*}(z) x_{n}(z)-p F\left(z, x_{n}(z)\right)}{\left|u_{n}^{*}(z)\right|^{1+1 / r}} \frac{\left|u_{n}^{*}(z)\right|}{\left\|x_{n}\right\|_{1, p}^{1+1 / r}} d z .
\end{aligned}
$$

Because of hypothesis (H)(ii) we have for some $c_{6}>0$

$$
\begin{aligned}
\left|\int_{\left\{0 \leq x_{n}<\eta\right\}} \frac{u_{n}^{*}(z) x_{n}(z)-p F\left(z, x_{n}(z)\right)}{\left\|x_{n}\right\|_{1, p}^{1+1 / r}} d z\right| \leq \frac{c_{6}}{\left\|x_{n}\right\|_{1, p}^{1 / r}} \stackrel{n \rightarrow \infty}{\longrightarrow} 0, \\
\Longrightarrow \int_{\left\{x_{n} \geq \eta\right\}} \frac{u_{n}^{*}(z) x_{n}(z)-p F\left(z, x_{n}(z)\right)}{\left|u_{n}^{*}(z)\right|^{1+1 / r}} \\
\quad \times \frac{\left|u_{n}^{*}(z)\right|^{1+1 / r}}{\left\|x_{n}\right\|_{1, p}^{1+1 / r}} d z \stackrel{n \rightarrow \infty}{\longrightarrow} 0 \quad \text { (from (3.13) and (3.15)), } \\
\left.\Longrightarrow \int_{\left\{x_{n} \geq \eta\right\}} \frac{\left|u_{n}^{*}(z)\right|^{1+1 / r}}{\left\|x_{n}\right\|_{1, p}^{1+1 / r}} d z \stackrel{n \rightarrow \infty}{\longrightarrow} 0 \quad \text { (see hypothesis }(\mathrm{H})(\mathrm{v})\right) .
\end{aligned}
$$

Moreover, using once again hypothesis (H)(ii) and the fact that $f_{1}\left(z, x_{n}(z)\right) \leq$ $u_{n}^{*}(z) \leq f_{2}\left(z, x_{n}(z)\right)$ a.e. on $Z$, we have

$$
\int_{\left\{0 \leq x_{n}<\eta\right\}} \frac{\left|u_{n}^{*}(z)\right|^{1+1 / r}}{\left\|x_{n}\right\|_{1, p}^{1+1 / r}} d z \stackrel{n \rightarrow \infty}{\longrightarrow} 0 .
$$

Finally since $\left|u_{n}^{*}(z)\right| \leq a_{1}(z)$ a.e. on $\left\{x_{n}<0\right\}$ and $a_{1} \in L^{\infty}(Z)$ (see hypothesis (H)(ii)), we have

$$
\begin{gathered}
\int_{\left\{x_{n}<0\right\}} \frac{\left|u_{n}^{*}(z)\right|^{1+1 / r}}{\left\|x_{n}\right\|_{1, p}^{1+1 / r}} d z \leq \int_{\left\{x_{n}<0\right\}} \frac{a_{1}(z)^{1+1 / r}}{\left\|x_{n}\right\|_{1, p}^{1+1 / r}} d z \stackrel{n \rightarrow \infty}{\longrightarrow} 0, \\
\Longrightarrow \frac{u_{n}^{*}}{\left\|x_{n}\right\|_{1, p}} \stackrel{n \rightarrow \infty}{\longrightarrow} 0 \text { in } L^{1+1 / r}(Z) .
\end{gathered}
$$

If $\theta=1+1 / r$, then $\theta^{\prime}=r+1 \leq p^{*},\left(1 / \theta+1 / \theta^{\prime}=1\right)$, and so by the Sobolev embedding theorem we have that $W_{0}^{1, p}(Z)$ is embedded continuously in $L^{\theta^{\prime}}(Z)$, hence $L^{\theta}(Z)=L^{\theta^{\prime}}(Z)^{*}$ is embedded continuously in $W^{-1, q}(Z)=W_{0}^{1, p}(Z)^{*}$. So $u_{n}^{*} /\left\|x_{n}\right\|_{1, p}$ $\stackrel{n \rightarrow \infty}{\longrightarrow} 0$ in $W^{-1, q}(Z)$. 
Let $y_{n}=x_{n} /\left\|x_{n}\right\|_{1, p}, n \geq 1$. Since $\left\|y_{n}\right\|_{1, p}=1, n \geq 1$, by passing to a subsequence if necessary, we may assume that $y_{n} \stackrel{w}{\longrightarrow} y$ in $W_{0}^{1, p}(Z), y_{n} \rightarrow y$ in $L^{p}(Z)$ (because $W_{0}^{1, p}(Z)$ is embedded compactly in $\left.L^{p}(Z)\right), y_{n} \stackrel{w}{\longrightarrow} y$ in $L^{\theta^{\prime}}(Z)$ (because $W_{0}^{1, p}(Z)$ is embedded continuously in $\left.L^{\theta^{\prime}}(Z)\right), y_{n}(z) \rightarrow y(z)$ a.e. on $Z$ as $n \rightarrow \infty$ and $\left|y_{n}(z)\right| \leq$ $h(z)$ a.e. on $Z$ with $h \in L^{p}(Z)$.

Recall that $m\left(x_{n}\right) \rightarrow 0$ and so

$$
\left\langle A\left(x_{n}\right), y_{n}-y\right\rangle-\lambda_{1}\left(J\left(x_{n}\right), y_{n}-y\right)_{p q}-\left\langle u_{n}^{*}, y_{n}-y\right\rangle \leq \varepsilon_{n}\left\|y_{n}-y\right\|_{1, p}, \quad n \geq 1 .
$$

Dividing by $\left\|x_{n}\right\|_{1, p}^{p-1}$, we obtain

$$
\left\langle A\left(y_{n}\right), y_{n}-y\right\rangle-\lambda_{1}\left(J\left(y_{n}\right), y_{n}-y\right)_{p q}-\left\langle\frac{u_{n}^{*}}{\left\|x_{n}\right\|_{1, p}}, \frac{y_{n}-y}{\left\|x_{n}\right\|_{1, p}^{p-2}}\right\rangle \leq \frac{\varepsilon_{n}}{\left\|x_{n}\right\|_{1, p}^{p-1}}\left\|y_{n}-y\right\|_{1, p} \text {. }
$$

We know that $u_{n}^{*} /\left\|x_{n}\right\|_{1, p} \stackrel{n \rightarrow \infty}{\longrightarrow} 0$ in $W^{-1, q}(Z)$, so $\left\langle u_{n}^{*} /\left\|x_{n}\right\|_{1, p},\left(y_{n}-y\right) /\left\|x_{n}\right\|_{1, p}^{p-2}\right\rangle$ $\stackrel{n \rightarrow \infty}{\longrightarrow} 0$. Moreover, $\left(J\left(y_{n}\right), y_{n}-y\right)_{p q} \stackrel{n \rightarrow \infty}{\longrightarrow} 0$. Thus finally we have

$$
\varlimsup\left\langle A\left(y_{n}\right), y_{n}-y\right\rangle \leq 0 \text {. }
$$

But $A$ being maximal monotone, is generalized pseudomonotone (cf. Hu and Papageorgiou [15, Definition III.6.2 and Remark III.6.3, page 365]). So we have

$$
A\left(y_{n}\right) \stackrel{w}{\longrightarrow} A(y) \quad \text { in } W^{-1, q}(Z) \text { as } n \longrightarrow \infty
$$

and $\left\langle A\left(y_{n}\right), y_{n}\right\rangle \rightarrow\langle A(y), y\rangle \Rightarrow\left\|D y_{n}\right\|_{p} \rightarrow\|D y\|_{p}$.

Since $D y_{n} \stackrel{w}{\longrightarrow} D y$ in $L^{p}\left(Z, \mathbb{R}^{N}\right)$ and the latter is uniformly convex, from the KadecKlee property we infer that $D y_{n} \rightarrow D y$ in $L^{p}\left(Z, \mathbb{R}^{N}\right)$, hence $y_{n} \rightarrow y$ in $W_{0}^{1, p}(Z)$ and so $\|y\|_{1, p}=1$; that is, $y \neq 0$ (an alternative proof of this can be based on the continuity of $\left.A^{-1}\right)$.

Again from the choice of the sequence $\left\{x_{n}\right\}_{n \geq 1}$, we have that for every $u \in$ $W_{0}^{1, p}(Z)$

$$
\left|\left\langle A\left(x_{n}\right), u\right\rangle-\lambda_{1}\left(J\left(x_{n}\right), u\right)_{p q}-\int_{Z} u_{n}^{*}(z) u(z) d z\right| \leq \varepsilon_{n}\|u\|_{1, p} .
$$

Dividing by $\left\|x_{n}\right\|_{1, p}^{p-1}$, we obtain

$$
\begin{gathered}
\left|\left\langle A\left(y_{n}\right), u\right\rangle-\lambda_{1}\left(J\left(y_{n}\right), u\right)_{p q}-\int_{Z} \frac{u_{n}^{*}(z)}{\left\|x_{n}\right\|_{1, p}} \frac{u(z)}{\left\|x_{n}\right\|_{1, p}^{p-2}} d z\right| \leq \frac{\varepsilon_{n}}{\left\|x_{n}\right\|_{1, p}^{p-1}}\|u\|_{1, p} \\
\Longrightarrow\langle A(y), u\rangle=\lambda_{1}(J(y), u)_{p q} \quad \forall u \in W_{0}^{1, p}(Z) \\
\Longrightarrow \int_{Z}\|D y(z)\|^{p-2}(D y(z), D u(z))_{\mathbb{R}^{N}} d z \\
=\lambda_{1} \int_{Z}|y(z)|^{p-2} y(z) u(z) d z \quad \forall u \in W_{0}^{1, p}(Z) \\
\Longrightarrow\left\langle-\operatorname{div}\left(\|D y\|^{p-2} D y\right), u\right\rangle=\lambda_{1}\left(|y|^{p-2} y, u\right)_{p q} \quad \forall u \in W_{0}^{1, p}(Z) .
\end{gathered}
$$


(Note that $\operatorname{div}\left(\|D y\|^{p-2} D y\right) \in W^{-1, q}(Z)$; recall the representation theorem for the elements in $W^{-1, q}(Z)=W_{0}^{1, p}(Z)^{*}$, see, for example, Adams [1, Theorem 3.10, page 50].) So we infer

$$
\begin{aligned}
-\operatorname{div}\left(\|D y(z)\|^{p-2} D y(z)\right) & =\lambda_{1}|y(z)|^{p-2} y(z) \quad \text { a.e. on } Z,\left.y\right|_{\Gamma}=0 \\
& \Longrightarrow y= \pm u_{1} .
\end{aligned}
$$

Suppose without any loss of generality that $y=u_{1}$ (the proof for the case $y=-u_{1}$ is similar). Since $u_{1}(z)>0$ for all $z \in Z$, we have that $x_{n}(z) \stackrel{n \rightarrow \infty}{\longrightarrow} \infty$ a.e. on $Z$. Recall that

$$
\begin{aligned}
&\left|\left\langle x_{n}^{*}, x_{n}\right\rangle\right| \leq \varepsilon_{n} \quad(\text { see }(3.7)) \\
& \Longrightarrow-\varepsilon_{n} \leq\left\langle A\left(x_{n}\right), x_{n}\right\rangle-\lambda_{1}\left(J\left(x_{n}\right), x_{n}\right)_{p q}-\int_{Z} u_{n}^{*}(z) x_{n}(z) d z \leq \varepsilon_{n} \\
& \Longrightarrow-\varepsilon_{n} \leq\left\|D x_{n}\right\|_{p}^{p}-\lambda_{1}\left\|x_{n}\right\|_{p}^{p}-\int_{Z} u_{n}^{*}(z) x_{n}(z) d z \leq \varepsilon_{n} .
\end{aligned}
$$

By virtue of hypothesis $(\mathrm{H})(\mathrm{v})$ we have that $\int_{Z} u_{n}^{*}(z) x_{n}(z) d z \stackrel{n \rightarrow \infty}{\longrightarrow} 0$. So we infer that $\left\|D x_{n}\right\|_{p}^{p}-\lambda_{1}\left\|x_{n}\right\|_{p}^{p} \stackrel{n \rightarrow \infty}{\longrightarrow} 0$. From the choice of the sequence $\left\{x_{n}\right\}_{n \geq 1} \subseteq W_{0}^{1, p}(Z)$, we have that $R\left(x_{n}\right) \stackrel{n \rightarrow \infty}{\longrightarrow} c$. So given $\varepsilon>0$ we can find $n_{0}=n_{0}(\varepsilon) \geq 1$ such that for $n \geq n_{0}$ we have

$$
\begin{aligned}
c-\varepsilon & \leq R\left(x_{n}\right) \leq c+\varepsilon \\
& \Longrightarrow c-\varepsilon \leq \frac{1}{p}\left\|D x_{n}\right\|_{p}^{p}-\frac{\lambda_{1}}{p}\left\|x_{n}\right\|_{p}^{p}-\int_{Z} F\left(z, x_{n}(z)\right) d z \leq c+\varepsilon .
\end{aligned}
$$

Passing to the limit as $n \rightarrow \infty$, we obtain

$$
c-\varepsilon \leq-\int_{Z} F_{+}(z) d z \leq c+\varepsilon .
$$

Let $\varepsilon \downarrow 0$ to conclude that $c=-\int_{Z} F_{+}(z) d z$, a contradiction. This proves the claim.

Because of the claim and by passing to a subsequence if necessary, we may assume that $x_{n} \stackrel{w}{\longrightarrow} x$ in $W_{0}^{1, p}(Z)$ as $n \rightarrow \infty$. Also since $f_{1}\left(z, x_{n}(z)\right) \leq u_{n}^{*}(z) \leq f_{2}\left(z, x_{n}(z)\right)$ a.e. on $Z$, by virtue of hypothesis (H)(ii) we have that $\left\{u_{n}^{*}\right\}_{n \geq 1} \subseteq L^{\sigma^{\prime}}(Z)$ is bounded. Therefore,

$$
\begin{gathered}
\left\langle A\left(x_{n}\right), x_{n}-x\right\rangle=\left\langle x_{n}^{*}, x_{n}-x\right\rangle-\lambda_{1}\left(J\left(x_{n}\right), x_{n}-x\right)_{p q}-\left(u_{n}^{*}, x_{n}-x\right)_{\sigma \sigma^{\prime}} \\
\Longrightarrow \varlimsup \lim \left\langle A\left(x_{n}\right), x_{n}-x\right\rangle=0 .
\end{gathered}
$$

But $A$ being maximal monotone, is generalized pseudomonotone (see $\mathrm{Hu}$ and Papageorgiou [15, Definition III.6.2 and Remark III.6.3, page 365]). So we have $\left\langle A\left(x_{n}\right), x_{n}\right\rangle \stackrel{n \rightarrow \infty}{\longrightarrow}\langle A(x), x\rangle \Rightarrow\left\|D x_{n}\right\|_{p} \stackrel{n \rightarrow \infty}{\longrightarrow}\|D x\|_{p}$. On the other hand, we know that $D x_{n} \stackrel{w}{\longrightarrow} D x$ in $L^{p}\left(Z, \mathbb{R}^{n}\right)$ and $L^{p}\left(Z, \mathbb{R}^{N}\right)$ has the Kadec-Klee property (being uniformly convex). So $D x_{n} \stackrel{n \rightarrow \infty}{\longrightarrow} D x$ in $L^{p}\left(Z, \mathbb{R}^{N}\right)$, hence $x_{n} \stackrel{n \rightarrow \infty}{\longrightarrow} x$ in $W_{0}^{1, p}(Z)$. 
The next proposition allows the use of Theorem 2.1 (see also Proposition 3 of Kourogenis and Papageorgiou [16]).

Proposition 3.5. If hypotheses $(H)$ hold, then we can find $\beta_{1}, \beta_{2}>0$ and $p<v \leq p^{*}$ such that $R(x) \geq \beta_{1}\|x\|_{1, p}^{p}-\beta_{2}\|x\|_{1, p}^{v}$ for all $x \in W_{0}^{1, p}(Z)$.

Proof. By virtue of hypothesis (H)(vi) we can find $\mu<-\lambda_{1}$ and $\delta>0$ such that for almost all $z \in Z$ and all $|x| \leq \delta$, we have

$$
F(z, x) \leq \frac{1}{p} \mu|x|^{p}
$$

On the other hand, from hypothesis $(\mathrm{H})(\mathrm{ii})$ we have

$$
|F(z, x)| \leq a_{3}(z)|x|+\frac{c_{2}}{p}|x|^{\sigma} \text { a.e. on } Z \forall x \in \mathbb{R},
$$

with $a_{3}(z)=\max \left\{a_{1}(z), a_{2}(z)\right\} \in L^{\infty}(Z)$.

Therefore, we can find $\gamma>0$ large enough and $p<v \leq p^{*}$ such that the inequality

$$
F(z, x) \leq \frac{1}{p} \mu|x|^{p}+\gamma|x|^{\nu} \quad \text { a.e. on } Z \forall x \in \mathbb{R}
$$

holds.

Then for every $x \in W_{0}^{1, p}(Z)$ we have

$$
\begin{aligned}
R(x) & =\frac{1}{p}\|D x\|_{p}^{p}-\frac{\lambda_{1}}{p}\|x\|_{p}^{p}-\int_{Z} F(z, x(z)) d z \\
& \geq \frac{1}{p}\|D x\|_{p}^{p}-\frac{\lambda_{1}}{p}\|x\|_{p}^{p}-\frac{1}{p} \mu\|x\|_{p}^{p}-\gamma\|x\|_{v}^{v} \\
& \geq \frac{1}{p}\|D x\|_{p}^{p}-\frac{1}{p}\left(\lambda_{1}+\mu\right)\|x\|_{p}^{p}-\gamma\|x\|_{v}^{v} \\
& \geq \frac{1}{p}\left(-\frac{\mu}{\lambda_{1}}\right)\|D x\|_{p}^{p}-\gamma\|x\|_{\nu}^{v} .
\end{aligned}
$$

Note that $(1 / p)\left(-\mu / \lambda_{1}\right)=\hat{\beta}_{1}>0$. From Poincaré's inequality and since $W_{0}^{1, p}(z)$ is continuously embedded in $L^{v}(Z)$ (recall that $v \leq p^{*}$ ), we can find $\beta_{1}, \beta_{2}>0$ such that

$$
R(x) \geq \beta_{1}\|x\|_{1, p}^{p}-\beta_{2}\|x\|_{1, p}^{v} \quad \forall x \in W_{0}^{1, p}(Z) .
$$

\section{Multiplicity result}

Now we have the necessary tools to state and prove a multiplicity theorem for problem (2.8). 
THEOREM 4.1. If hypotheses $(H)$ hold, then problem (2.8) has at least three nontrivial solutions.

Proof. Let $U^{ \pm}=\left\{x \in W_{0}^{1, p}(Z): x= \pm t u_{1}+v, t>0, v \in V\right\}$. We show that $R(\cdot)$ attains its infimum on both open sets $U^{+}$and $U^{-}$. To this end let $m_{+}=\inf [R(x): x \in$ $\left.U^{+}\right]=\inf \left[R(x): x \in \bar{U}^{+}\right]$(since $R(\cdot)$ is locally Lipschitz). Let

$$
\bar{R}(x)= \begin{cases}R(x) & \text { if } x \in \bar{U}^{+} \\ +\infty & \text { otherwise }\end{cases}
$$

Evidently $\bar{R}(\cdot)$ is a lower semicontinuous function which is bounded below (see Proposition 3.1). Apply Theorem 2.2 with $x_{0}=0, h(r)=r, \varepsilon=\varepsilon_{n}^{2}$, where $\varepsilon_{n} \downarrow 0$ and $\lambda=\varepsilon_{n}$. Then noting Propositions 3.2 and 3.3, we can produce a sequence $\left\{x_{n}\right\}_{n \geq 1} \subseteq U^{+}$ such that $R\left(x_{n}\right) \downarrow m_{+}$(minimizing sequence) and

$$
\begin{aligned}
\bar{R}\left(x_{n}\right) \leq \bar{R}(u)+ & \frac{\varepsilon_{n}}{\left(1+\left\|x_{n}\right\|_{1, p}\right)}\left\|x_{n}-u\right\|_{1, p} \quad \forall u \in W_{0}^{1, p}(Z) \\
& \Longrightarrow-\frac{\varepsilon_{n}}{\left(1+\left\|x_{n}\right\|_{1, p}\right)}\left\|x_{n}-u\right\|_{1, p} \leq \bar{R}(u)-\bar{R}\left(x_{n}\right) .
\end{aligned}
$$

Let $u=x_{n}+t w$, with $t>0$ and $w \in W_{0}^{1, p}(Z)$. Because $x_{n} \in U^{+}$and $U^{+}$is open, we can find $\delta>0$ such that for $0 \leq t \leq \delta$ we have $x_{n}+t w \in U^{+}$. Hence we have

$$
\begin{aligned}
-\frac{\varepsilon_{n}}{\left(1+\left\|x_{n}\right\|_{1, p}\right)}\|w\|_{1, p} \leq & \frac{R\left(x_{n}+t w\right)-R\left(x_{n}\right)}{t} \quad \text { for } 0<t \leq \delta \\
& \Longrightarrow-\frac{\varepsilon_{n}}{\left(1+\left\|x_{n}\right\|_{1, p}\right)}\|w\|_{1, p} \leq R^{0}\left(x_{n} ; w\right) .
\end{aligned}
$$

Let $\psi_{n}(w)=\left(\left(1+\left\|x_{n}\right\|_{1, p}\right) / \varepsilon_{n}\right) R^{0}\left(x_{n} ; w\right)$. Then $\psi_{n}(\cdot)$ is sublinear, continuous with $\psi_{n}(0)=0$ and $-\|w\|_{1, p} \leq \psi_{n}(w)$ for all $w \in W_{0}^{1, p}(Z)$. We can apply Lemma 1.3 of Szulkin [20] and obtain $y_{n}^{*} \in W^{-1, q}(Z), n \geq 1$, such that $\left\|y_{n}^{*}\right\| \leq 1$ and $\left\langle y_{n}^{*}, w\right\rangle \leq$ $\psi_{n}(w)$ for all $w \in W_{0}^{1, p}(Z)$ and all $n \geq 1$. Then if $x_{n}^{*}=\varepsilon_{n} /\left(1+\left\|x_{n}\right\|_{1, p}\right) y_{n}^{*}$, we have $\left\langle x_{n}^{*}, w\right\rangle \leq R^{0}\left(x_{n} ; w\right)$ for all $w \in W_{0}^{1, p}(Z)$, hence $x_{n}^{*} \in \partial R\left(x_{n}\right), n \geq 1$. We have $\left(1+\left\|x_{n}\right\|_{1, p}\right) m\left(x_{n}\right) \leq\left(1+\left\|x_{n}\right\|\right)\left\|x_{n}^{*}\right\| \leq \varepsilon_{n}\left\|y_{n}^{*}\right\| \leq \varepsilon_{n} \stackrel{n \rightarrow \infty}{\longrightarrow} 0$. Note that because of hypothesis $(\mathrm{H})(\mathrm{vii}), m_{+}<-\int_{Z} F_{+}(z) d z$. So we can use Proposition 3.4 and assume that $x_{n} \rightarrow y_{1}$ in $W_{0}^{1, p}(Z)$ with $y_{1} \in \bar{U}^{+}$. Then $R\left(x_{n}\right) \stackrel{n \rightarrow \infty}{\longrightarrow} R\left(y_{1}\right)=m_{+}$. If $y_{1} \in \partial \bar{U}^{+}=V$, by Propositions 3.2 and 3.3 we have

$$
0 \leq R\left(y_{1}\right)=m_{+}<0,
$$

a contradiction. So $y_{1} \in U^{+}$, hence is a local minimum of $R(\cdot)$. This means that $0 \in \partial R\left(y_{1}\right)$. Similarly, working on $\bar{U}^{-}$we obtain $y_{2} \in U^{-}$such that $0 \in \partial R\left(y_{2}\right)$. Clearly $y_{2} \neq y_{1}$. 
By virtue of Proposition 3.5 we can find $0<\rho<\min \left\{\xi_{+}, \xi_{-}\right\}$such that $R_{\mid \partial B \rho}>0>$ $m \pm$. Thus we can apply Theorem 2.1 (with $y=\xi_{+} u_{1}$ or $y=\xi_{-} u_{1}$ ) and obtain $y_{3} \neq y_{1}$, $y_{3} \neq y_{2}, y_{3} \neq 0$ such that $0 \in \partial R\left(y_{3}\right)$.

Finally let $y=y_{k}, k=\{1,2,3\}$. Since $0 \in \partial R(y)$, we have

$$
A(y)-\lambda_{1}|y|^{p-2} y-u^{*}=0,
$$

for some $u^{*} \in \partial \psi(y)$ (hence $f_{1}(z, y(z)) \leq u^{*}(z) \leq f_{2}(z, y(z))$ a.e. on $Z$ ). Thus for every $u \in C_{0}^{\infty}(Z)$ we have

$$
\begin{aligned}
\langle A(y), u\rangle & -\lambda_{1}(J(y), u)_{p q}-\left(u^{*}, u\right)_{\sigma \sigma^{\prime}}=0 \\
& \Longrightarrow \int_{Z}\|D y(z)\|^{p-2}(D y(z), D u(z))_{\mathbb{R}^{N}} d z \\
& =\int_{Z}\left(u^{*}(z)+\lambda_{1}|y(z)|^{p-2} y(z)\right) u(z) d z
\end{aligned}
$$

Using the definition of the distributional derivative and since $\operatorname{div}\left(\|D y\|^{p-2} D y\right) \in$ $W^{-1, q}(Z)$ (see Adams [1, Theorem 3.10, page 50]), we have

$$
\left\langle-\operatorname{div}\left(\|D y\|^{p-2} D y\right), u\right\rangle-\lambda_{1}(J(y), u)_{p q}-\left(u^{*}, u\right)_{\sigma \sigma^{\prime}}=0 \quad \forall u \in C_{0}^{\infty}(Z) .
$$

Since $C_{0}^{\infty}(Z)$ is dense in $W_{0}^{1, p}(Z)$, we conclude that

$$
\begin{array}{r}
-\operatorname{div}\left(\|D y(z)\|^{p-2} D y(z)\right)-\lambda_{1}|y(z)|^{p-2} y(z)=u^{*}(z) \\
\text { a.e. on } Z,\left.y\right|_{\Gamma}=0,2 \leq p<\infty,
\end{array}
$$

with $u^{*}(z) \in \hat{f}(z, y(z))$ a.e. on $Z$. So $y$ is a solution of (2.8). Therefore $y_{1}, y_{2}$, and $y_{3}$ are three distinct nonzero solutions of (2.8).

Remark 4.2. If we drop hypothesis $(\mathrm{H})(\mathrm{vi})$, we can still have a third solution $y_{3}$, via the nonsmooth saddle point theorem (see Chang [10, Theorem 3.3, page 118]), provided $\xi_{+}=\left|\xi_{-}\right|$. Indeed Propositions 3.2, 3.3, and 3.4 allow the use of the nonsmooth saddle point theorem. However, in general we cannot guarantee that $y_{3} \neq 0$.

We conclude this paper with a result which highlights the difference between the nonsmooth (PS)-condition and the nonsmooth $C$-condition and also extends to the present nonsmooth setting a result well known for "smooth" functions.

It has been observed that in the differentiable case, the (PS)-condition implies coercivity for a functional which is bounded below. This was proved by Costa and Silva [12] (for a Frechet differentiable functional) and by Calkovic et al. [6] (for a Gateaux differentiable functional which is also lower semicontinuous). In the next proposition, we extend this result to the present nonsmooth case.

Proposition 4.3. If $Y$ is a Banach space, $\phi: Y \rightarrow \mathbb{R}$ is locally Lipschitz and bounded below and $c=\underline{\lim }_{\|y\| \rightarrow \infty} \phi(y)$ is finite, then there exists a sequence $\left\{x_{n}\right\}_{n \geq 1} \subseteq Y$ such that $\left\|x_{n}\right\| \rightarrow \infty, \phi\left(x_{n}\right) \rightarrow c$, and $m\left(x_{n}\right) \rightarrow 0$ as $n \rightarrow \infty$. 
Proof. We can find $\left\{y_{n}\right\}_{n \geq 1} \subseteq Y$ such that

$$
\phi\left(y_{n}\right) \leq c+\frac{1}{n}, \quad\left\|y_{n}\right\| \geq 2 n
$$

Let $\eta=\inf [\phi(y): y \in Y]$. Because $\phi(\cdot)$ is bounded below, $\eta$ is finite. Apply Theorem 2.2 with $h(r)=0, x_{0}=y_{n}, \varepsilon=\varepsilon_{n}=c+1 / n-\eta$ and $\lambda=\lambda_{n}=n$. We can find $x_{n} \in Y, n \geq 1$, such that

$$
\begin{gathered}
\phi\left(x_{n}\right) \leq \phi\left(y_{n}\right) \leq c+\frac{1}{n}, \\
\phi\left(x_{n}\right) \leq \phi(u)+\frac{\varepsilon_{n}}{n}\left\|x_{n}-u\right\| \quad \forall u \in Y, \\
\left\|x_{n}-y_{n}\right\| \leq n \quad \forall n \geq 1 .
\end{gathered}
$$

Let $u=x_{n}+t v$ with $t>0$ and $v \in Y$. Then we have

$$
-\xi_{n}\|v\| \leq \frac{\phi\left(x_{n}+t v\right)-\phi\left(x_{n}\right)}{t}
$$

where $\xi_{n}=\left(\varepsilon_{n} / n\right) \downarrow 0$ as $n \rightarrow \infty$. Letting $t \downarrow 0$ we obtain

$$
-\xi_{n}\|v\| \leq \phi^{0}\left(x_{n} ; v\right) \quad \forall n \geq 1 \text { and all } v \in Y
$$

Let $\psi_{n}(v)=\left(1 / \xi_{n}\right) \phi^{0}\left(x_{n} ; v\right)$. Then $\psi(\cdot)$ is sublinear, continuous with $\psi(0)=0$ and $-\|v\| \leq \psi_{n}(v)$ for all $v \in Y$. Invoking Lemma 1.3 of Szulkin [20], we can find $y_{n}^{*} \in Y^{*}$, $n \geq 1$, such that $\left\|y_{n}^{*}\right\| \leq 1$ and $\left(y_{n}^{*}, v\right) \leq \psi_{n}(v)$ for all $n \geq 1$ and all $v \in Y$. Then if $x_{n}^{*}=\xi_{n} y_{n}^{*}$, we have

$$
\begin{aligned}
\left(x_{n}^{*}, v\right) & \leq \phi^{0}\left(x_{n}, v\right) \quad \forall n \geq 1 \text { and all } v \in Y, \\
& \Longrightarrow x_{n}^{*} \in \partial \phi\left(x_{n}\right) \quad \forall n \geq 1 .
\end{aligned}
$$

Hence $m\left(x_{n}\right) \leq\left\|x_{n}^{*}\right\| \leq \xi_{n} \stackrel{n \rightarrow \infty}{\longrightarrow} 0$. Also

$$
\begin{gathered}
\left\|x_{n}\right\| \geq\left\|y_{n}\right\|-\left\|x_{n}-y_{n}\right\| \geq 2 n-n=n \longrightarrow+\infty \\
\Longrightarrow \phi\left(x_{n}\right) \stackrel{n \rightarrow \infty}{\longrightarrow} c .
\end{gathered}
$$

Therefore $\left\{x_{n}\right\}_{n \geq 1} \subseteq Y$ is the desired sequence.

An immediate consequence of this proposition is the following corollary.

Corollary 4.4. If $Y$ is a Banach space, $\phi: Y \rightarrow \mathbb{R}$ is locally Lipschitz, bounded below and satisfies the nonsmooth (PS)-condition, then $\phi(\cdot)$ is coercive. 
134 Multiple solutions

\section{Acknowledgement}

The authors wish to express their gratitude to a very knowledgeable referee for his very helpful corrections and remarks that improved the paper considerably.

\section{References}

[1] R. A. Adams, Sobolev Spaces, vol. 65, Academic Press [A subsidiary of Harcourt Brace Jovanovich, Publishers], New York, 1975, Pure and Applied Mathematics. MR 56\#9247. Zbl 314.46030.

[2] S. Ahmad, Multiple nontrivial solutions of resonant and nonresonant asymptotically linear problems, Proc. Amer. Math. Soc. 96 (1986), no. 3, 405-409. MR 87m:35090. Zbl 634.35029.

[3] S. Ahmad, A. C. Lazer, and J. L. Paul, Elementary critical point theory and perturbations of elliptic boundary value problems at resonance, Indiana Univ. Math. J. 25 (1976), no. 10, 933-944. MR 55\#855. Zbl 351.35036.

[4] C. O. Alves, P. C. Carrião, and O. H. Miyagaki, Multiple solutions for a problem with resonance involving the p-Laplacian, Abstr. Appl. Anal. 3 (1998), no. 1-2, 191-201. MR 2000d:35062. Zbl 992.14822.

[5] A. Ambrosetti and P. H. Rabinowitz, Dual variational methods in critical point theory and applications, J. Functional Analysis 14 (1973), 349-381. MR 51\#6412. Zbl 273.49063.

[6] A. Anane, Simplicité et isolation de la première valeur propre du p-Laplacien avec poids [Simplicity and isolation of the first eigenvalue of the p-Laplacian with weight], C. R. Acad. Sci. Paris Sér. I Math. 305 (1987), no. 16, 725-728 (French). MR 89e:35124. Zbl 633.35061.

[7] A. Anane and N. Tsouli, On the second eigenvalue of the p-Laplacian, Nonlinear Partial Differential Equations (Fès, 1994), Pitman Res. Notes Math. Ser., vol. 343, Longman, Harlow, 1996, pp. 1-9. MR 97k:35190. Zbl 854.35081.

[8] P. Bartolo, V. Benci, and D. Fortunato, Abstract critical point theorems and applications to some nonlinear problems with "strong" resonance at infinity, Nonlinear Anal. 7 (1983), no. 9, 981-1012. MR 85c:58028. Zbl 522.58012.

[9] G. Cerami, Un criterio di esistenza per i punti critici su varieta' illimitate [An existence criterion for the critical points on unbounded manifolds], Istit. Lombardo Accad. Sci. Lett. Rend. A 112 (1978), no. 2, 332-336 (Italian). MR 81k:58021. Zbl 436.58006.

[10] K. C. Chang, Variational methods for nondifferentiable functionals and their applications to partial differential equations, J. Math. Anal. Appl. 80 (1981), no. 1, 102-129. MR 82h:35025. Zbl 487.49027.

[11] F. H. Clarke, Optimization and Nonsmooth Analysis, Canadian Mathematical Society Series of Monographs and Advanced Texts, John Wiley \& Sons, New York, 1983, A WileyInterscience Publication. MR 85m:49002. Zbl 582.49001.

[12] D. G. Costa and E. A. de B. e Silva, The Palais-Smale condition versus coercivity, Nonlinear Anal. 16 (1991), no. 4, 371-381. MR 92i:58033. Zbl 733.49011.

[13] J. V. A. Goncalves and O. H. Miyagaki, Multiple nontrivial solutions of semilinear strongly resonant elliptic equations, Nonlinear Anal. 19 (1992), no. 1, 43-52. MR 93h:35064. Zbl 784.35032.

[14] _ Three solutions for a strongly resonant elliptic problem, Nonlinear Anal. 24 (1995), no. 2, 265-272. MR 96e:35046. Zbl 821.35052.

[15] S. Hu and N. S. Papageorgiou, Handbook of Multivalued Analysis. Vol. I: Theory, Mathematics and its Applications, vol. 419, Kluwer Academic Publishers, Dordrecht, 1997. MR 98k:47001. Zbl 887.47001. 
[16] N. C. Kourogenis and N. S. Papageorgiou, Discontinuous quasilinear elliptic problems at resonance, Colloq. Math. 78 (1998), no. 2, 213-223. MR 2000d:35056. Zbl 920.35061.

[17] Multiple solutions for nonlinear discontinuous elliptic problems near resonance, Colloq. Math. 81 (1999), no. 1, 89-99. MR 2000i:35045. Zbl 933.35152.

[18] E. Landesman, S. Robinson, and A. Rumbos, Multiple solutions of semilinear elliptic problems at resonance, Nonlinear Anal. 24 (1995), no. 7, 1049-1059. MR 96e:35049. Zbl 832.35048.

[19] P. H. Rabinowitz, Minimax Methods in Critical Point Theory with Applications to Differential Equations, CBMS Regional Conference Series in Mathematics, vol. 65, American Mathematics Society, Rhode Island, 1986. MR 87j:58024. Zbl 609.58002.

[20] A. Szulkin, Minimax principles for lower semicontinuous functions and applications to nonlinear boundary value problems, Ann. Inst. H. Poincaré Anal. Non Linéaire 3 (1986), no. 2, 77-109. MR 87f:49021. Zbl 612.58011.

[21] K. Thews, Nontrivial solutions of elliptic equations at resonance, Proc. Roy. Soc. Edinburgh Sect. A 85 (1980), no. 1-2, 119-129. MR 81c:35054. Zbl 431.35040.

[22] P. Tolksdorf, Regularity for a more general class of quasilinear elliptic equations, J. Differential Equations 51 (1984), no. 1, 126-150. MR 85g:35047. Zbl 522.35018.

[23] J. R. Ward, Jr., Applications of critical point theory to weakly nonlinear boundary value problems at resonance, Houston J. Math. 10 (1984), no. 2, 291-305. MR 85i:35064. Zbl 594.35037.

[24] C.-K. Zhong, On Ekeland's variational principle and a minimax theorem, J. Math. Anal. Appl. 205 (1997), no. 1, 239-250. MR 98a:58034. Zbl 870.49015.

Nikolaos C. Kourogenis: National Technical University, Department of Mathematics, Zografou Campus, Athens 157 80, Greece

Nikolaos S. Papageorgiou: National Technical University, Department of Mathematics, Zografou Campus, Athens 157 80, Greece

E-mail address: npapg@math.ntua.gr 


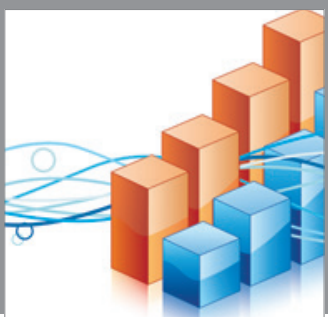

Advances in

Operations Research

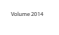

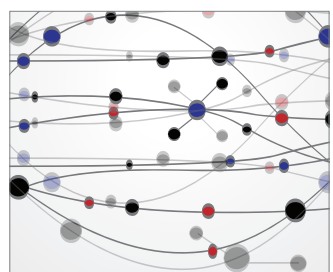

\section{The Scientific} World Journal
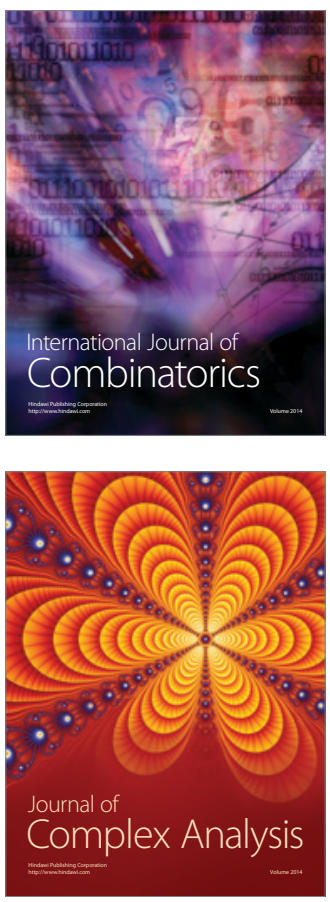

International Journal of

Mathematics and

Mathematical

Sciences
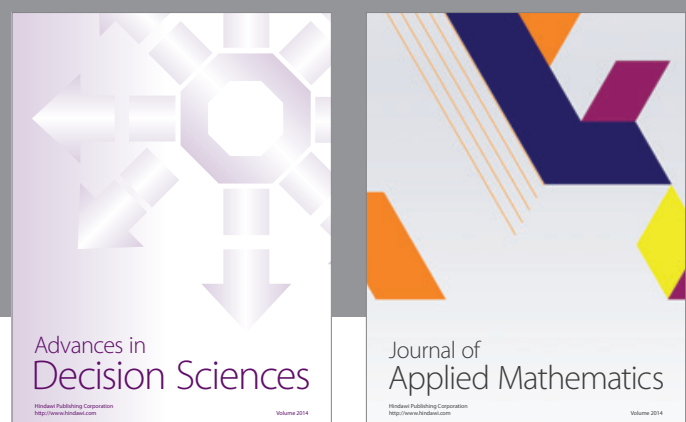

Journal of

Applied Mathematics
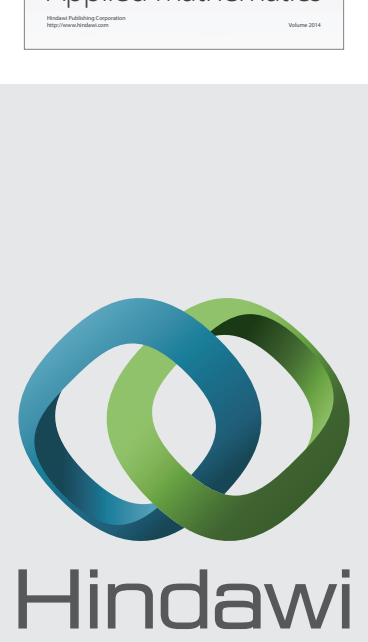

Submit your manuscripts at http://www.hindawi.com
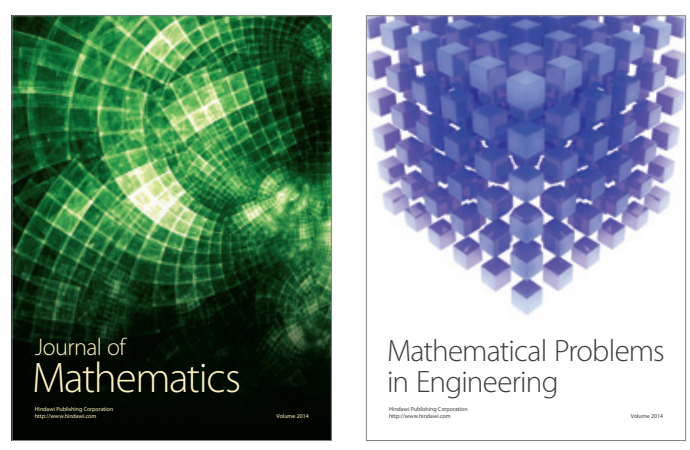

Mathematical Problems in Engineering
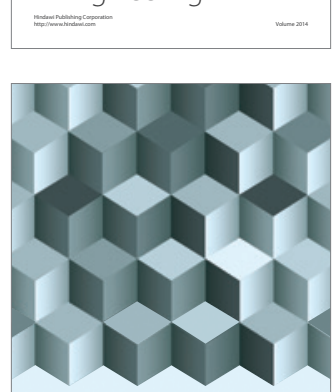

Journal of

Function Spaces
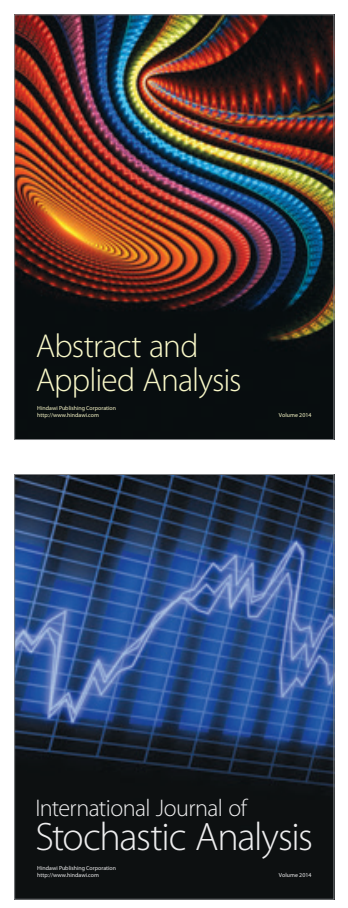

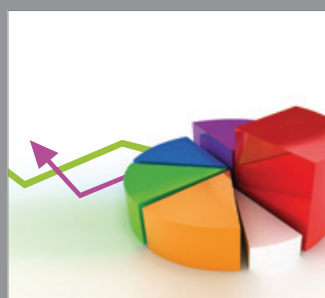

ournal of

Probability and Statistics

Promensencen
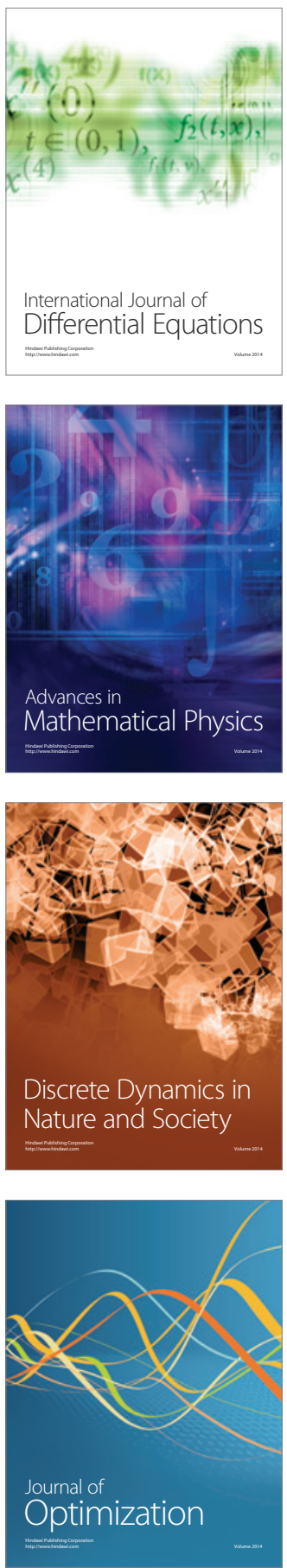